\title{
Magnetohydrodynamic simulation of the interaction between interplanetary strong shock and magnetic cloud and its consequent geoeffectiveness
}

Ming Xiong, ${ }^{1}$ Huinan Zheng, ${ }^{1}$ Yuming Wang, ${ }^{1}$ and Shui Wang ${ }^{1}$

Received 28 December 2005; revised 26 April 2006; accepted 2 May 2006; published 19 August 2006.

[1] Numerical studies have been performed to interpret the observed "shock overtaking magnetic cloud (MC)" event by a 2.5 dimensional magnetohydrodynamic (MHD) model in the heliospheric meridional plane. Results of an individual MC simulation show that the MC travels with a constant bulk flow speed. The MC is injected with a very strong inherent magnetic field over that in the ambient flow and expands rapidly in size initially. Consequently, the diameter of the $\mathrm{MC}$ increases in an asymptotic speed while its angular width contracts gradually. Meanwhile, simulations of MC-shock interaction are also presented, in which both a typical MC and a strong fast shock emerge from the inner boundary and propagate along the heliospheric equator, separated by an appropriate interval. The results show that the shock first catches up with the preceding $\mathrm{MC}$, then penetrates through the $\mathrm{MC}$, and finally merges with the MC-driven shock into a stronger compound shock. The morphologies of shock front in interplanetary space and MC body behave as a central concave and a smooth arc, respectively. The compression and rotation of the magnetic field serve as an efficient mechanism to cause a large geomagnetic storm. The MC is highly compressed by the overtaking shock. Contrarily, the transport time of the incidental shock influenced by the MC depends on the interval between their commencements. Maximum geoeffectiveness results from when the shock enters the core of preceding $\mathrm{MC}$, which is also substantiated to some extent by a corresponding simplified analytic model. Quantified by the Dst index, the specific result is that the geoeffectiveness of an individual MC is largely enhanced with $80 \%$ increment in maximum by an incidental shock.

Citation: Xiong, M., H. Zheng, Y. Wang, and S. Wang (2006), Magnetohydrodynamic simulation of the interaction between interplanetary strong shock and magnetic cloud and its consequent geoeffectiveness, J. Geophys. Res., 111, A08105, doi:10.1029/2005JA011593.

\section{Introduction}

[2] Coronal mass ejection (CME) is one of the most frequently eruptive phenomena in the solar atmosphere, which causes significant changes in coronal structure accompanied by observable mass outflow. A great deal of CME observation data have been accumulated by such spacecraft as OSO-7, Skylab, P78-1, SMM, ISEE3, Helios, Yohkoh, SOHO, Ulysses, Wind, and ACE over the past 30 years. A typical CME is launched into interplanetary (IP) space with magnetic flux of $10^{23} \mathrm{Mx}$ and plasma mass of $10^{16} \mathrm{~g}$ [Gosling, 1990; Webb et al., 1994]. The "solar flare myth" that CMEs have no fundamental association (in terms of cause and effect) with flares

\footnotetext{
${ }^{1}$ Chinese Academy of Sciences Key Laboratory for Basic Plasma Physics, School of Earth and Space Sciences, University of Science and Technology of China, Hefei, China.

Copyright 2006 by the American Geophysical Union. 0148-0227/06/2005JA011593\$09.00
}

[Gosling, 1993; Gosling and Hundhausen, 1995] is quite controversial [e.g., Svestka, 1995; Dryer, 1996]. More favorable is the equal importance of $\mathrm{CME}$ and flare concerning the source of IP transient disturbances and nonrecurrent geomagnetic storms [Dryer, 1996]. Statistical research shows that nearly half of all CMEs form magnetic clouds (MCs) in IP space [Klein and Burlaga, 1982; Cane et al., 1997]. MCs are of great concern in the space community because their regular magnetic field with large southward magnetic component always leads to a geomagnetic storm. The characteristics of MCs, as defined by Burlaga et al. [1981], are enhanced magnetic field, smooth rotation of the magnetic field, low proton temperature, and a low ratio of proton thermal to magnetic pressure $\beta_{p}$. Many studies modeled an MC by an ideal local cylinder with a force-free field [e.g., Goldstein, 1983; Burlaga, 1988; Farrugia et al., 1993; Kumar and Rust, 1996; Osherovich and Burlaga, 1997], though in a real situation, an MC should probably be a curved looplike structure with its feet connecting to the solar surface 
[Larson et al., 1997]. Numerical simulations have been carried out to investigate the behavior of isolated loop-like MCs with various magnetic field strengths, axis orientations, and speeds, on the basis of the flux rope model [e.g., Vandas and Odstrčil, 2000; Vandas etal., 1995, 1996a, 1996b, 1996c, 1997a, 1997b, 2002; Groth et al., 2000; Odstrčil et al., 2002; Schmidt and Cargill, 2003; Vandas, 2003; Manchester et al., 2004a, 2004b]. A great consistency was found between the in situ observations, theoretical analyses, and numerical simulations.

[3] Recent studies have focused on the existence of more complex structure, with less defined characteristics and a possible association with interactions among CMEs, shocks, $\mathrm{MCs}$, and corotating regions, such as complex ejecta [Burlaga et al., 2002], multiple MCs [Wang et al., 2002, 2003a], shock-penetrated MCs [Wang et al., 2003b; Berdichevsky et al., 2005], and so on. Most of the different physical phenomena, which are likely to occur during the propagation of a following faster CME overtaking a preceding slower CME, have been studied by both 2.5-dimensional (2.5-D) and three-dimensional (3-D) magnetohydrodynamic (MHD) numerical simulations: the interaction of a shock wave with an MC [Vandas et al., 1997a; Odstrčil et al., 2003], the interaction of two MCs [Odstrčil et al., 2003; Gonzalez-Esparza et al., 2004; Lugaz et al., 2005; Wang et al., 2005] and the acceleration of electrons associated with the shock-cloud interaction [Vandas and Odstrčil, 2004].

[4] The establishment of a space weather forecasting system is ongoing, as it is urgently needed by human civilization. A numerical MHD model may play a critical role in it [Dryer, 1998]. The IP medium is a pivotal node in cause-effect chains of solar-terrestrial transporting events. The correlation between Dst index and various IP parameters have been comprehensively studied [e.g., Burton et al., 1975; Vassiliadis et al., 1999] and applied in related numerical simulations [e.g., Vandas, 2003]. Moreover, some observation data-driven numerical models have already been applied in real-time "fearless forecasting": (1) the HAF (Hakamada-Akasofu-Fry) model based on kinetics [Fry et al., 2001, 2005; Intriligator et al., 2005; McKenna-Lawlor et al., 2005], (2) shock time of arrival (STOA) based on classical self-similarity blast wave theory [Smart and Shea, 1985], (3) the interplanetary shock propagation model (ISPM) based on 2.5-D MHD simulation [Smith and Dryer, 1990], and (4) an ensemble of the above three models [Dryer et al., 2001, 2004; McKenna-Lawlor et al., 2002; Fry et al., 2003, 2004].

[5] The observed "shock overtaking MC" event complicates IP dynamics. With a strong enough magnitude, a fast shock can propagate through a low $\beta \mathrm{MC}$ and survive as a discontinuity in the front part of the MC. It can even penetrate the $\mathrm{MC}$ and merge with the original MC-driven shock into a stronger compound shock. The evolution stages of MC-shock interaction detected by Wind and ACE spacecraft at $1 \mathrm{AU}$ may be reduced to two categories: (1) shock still in the MC, such as the 3-6 October 2000 and 5-7 November 2001 events [Wang et al., 2003b], and (2) shock ahead of the MC after completely penetrating it, such as the 20-21 March 2003 event [Berdichevsky et al., 2005]. Ruling out the possibility of weak shock dissipation in low $\beta$ MC plasma, the MC-shock compound at $1 \mathrm{AU}$ changes from category 1 to 2 , as their eruption interval decreases at solar corona. MC-shock interaction is also an IP cause of large geomagnetic storms [Wang et al., 2003b, 2003c]. Obviously, MC with a penetrating shock at various stages may result in different geoeffectiveness.

[6] In this paper, studies are presented to understand the dynamic process of the "shock overtaking MC" event and its effect on geomagnetic storm strength by numerical simulation on the basis of a 2.5-D ideal MHD model. A brief description of the MHD equations and the numerical scheme used to solve them, as well as the steady state solar wind, the MC configuration, and shock specification, is given in section 2. Simulation results of an individual MC are described in section 3. Results of MC-shock interaction are discussed and analyzed in section 4 . The geoeffectiveness of MC-shock interaction is discussed in section 5 . Finally, conclusions are summarized in section 6.

\section{Numerical MHD Model}

\subsection{Governing MHD Equations}

[7] The macroscope behavior of magnetized plasma can be well described with MHD equations by using the conservation laws, supplemented by the equation of state of fluids and divergence-free condition of the magnetic field. Since the IP magnetic field (IMF) corotates with the Sun, it is convenient to adopt a corotating coordinate system, in which the fluid velocity is parallel to the magnetic field. With the assumption of an ideal gas with a polytropic index $\gamma=5 / 3$ and neglecting the effects of viscosity, electrical resistivity, and thermal conduction, the ideal MHD equations are written as follows [cf. Jeffrey and Taniuti, 1964]:

$$
\begin{gathered}
\frac{\partial \rho}{\partial t}+\nabla \cdot(\rho \mathbf{v})=0 \\
\frac{\partial(\rho \mathbf{v})}{\partial t}+\nabla \cdot\left[\rho \mathbf{v v}+\left(p+\frac{1}{8 \pi} B^{2}\right) I-\frac{1}{4 \pi} \mathbf{B B}\right]=\mathbf{f} \\
\frac{\partial W}{\partial t}+\nabla \cdot\left[\left(\frac{\partial \mathbf{B}}{\gamma t}-\nabla \times(\mathbf{v} \times \mathbf{B})=0\right.\right. \\
\text { with } \left.\left.p+\frac{1}{2} \rho v^{2}\right) \mathbf{v}+\frac{1}{4 \pi} \mathbf{B} \times(\mathbf{v} \times \mathbf{B})\right]=\mathbf{f} \cdot \mathbf{v} \\
\mathbf{f}=-\rho\left[\frac{g R_{s}^{2}}{r^{2}} \frac{\mathbf{r}}{r}+\boldsymbol{\Omega} \times(\boldsymbol{\Omega} \times \mathbf{r})+2(\boldsymbol{\Omega} \times \mathbf{v})\right] \\
W=\frac{1}{2} \rho v^{2}+\frac{1}{8 \pi} B^{2}+\frac{p}{\gamma-1},
\end{gathered}
$$

where $\rho$ is the plasma mass density, $\mathbf{v}$ is the plasma velocity, $\mathbf{B}$ is the magnetic field, $p$ is the plasma pressure (sum of electron and proton pressures), $\Omega$ is the angular speed of solar rotation $\left(=2.9 \times 10^{-6} \mathrm{rad} / \mathrm{s}\right), I$ is the unit matrix, $R_{s}$ is the solar radius, and $g$ is the gravitational acceleration at the 
Table 1. Physical Parameters of Ambient Solar Wind at the Bottom $\left(25 R_{s}\right)$ and at Lagrangian Point $L 1\left(213 R_{s}\right)$

\begin{tabular}{llcc}
\hline Variable & \multicolumn{1}{c}{ Description } & $25 R_{S}$ & $213 R_{S}$ \\
\hline$N_{p}, \mathrm{~cm}^{-3}$ & proton number density & 550 & 8 \\
$v_{r}, \mathrm{~km} / \mathrm{s}$ & radial speed & 375 & 452 \\
$B, \mathrm{nT}$ & magnetic field strength & 400 & 6.4 \\
$\beta$ & thermal to magnetic pressure ratio & 0.23 & 0.93 \\
$T_{p}, 10^{5} \mathrm{~K}$ & proton temperature & 9.6 & 0.7 \\
$c_{f}, \mathrm{~km} / \mathrm{s}$ & radial fast characteristic speed & 372 & 61 \\
\hline
\end{tabular}

solar surface. Equations (1)-(4) are expressed in a spherical coordinate system $(r, \theta, \varphi)$, dealing with 2.5 -D problems in the meridional plane. Namely, the partial derivatives of all dependent variables with respect to azimuthal angle $\varphi$ are zero.

\subsection{Computational Techniques}

[8] The mathematical connotation of a shock overtaking the MC belongs to high-resolution problems for the interaction between discontinuity and complex smooth structure. A total variation diminishing (TVD) scheme, a shockcapturing method, is applied to numerically solve MHD equations [Harten, 1983; Ryu and Jones, 1995] and possesses a formal accuracy of the second order in smooth flow regions except at extreme points. An eight-wave model [Powell et al., 1995] is adopted to guarantee divergencefree condition of the magnetic field.

[9] Furthermore, the magnetic flux function $\psi$ is introduced to ensure the accuracy of the magnetic field in the region near the shock front and the $\mathrm{MC}$, which satisfies

$$
\frac{\partial \psi}{\partial t}+v_{r} \frac{\partial \psi}{\partial r}+\frac{v_{\theta}}{r} \frac{\partial \psi}{\partial \theta}=0
$$

with

$$
\mathbf{B}=\left(\frac{1}{r^{2} \sin \theta} \frac{\partial \psi}{\partial \theta},-\frac{1}{r \sin \theta} \frac{\partial \psi}{\partial r}, B_{\varphi}\right)
$$

Equation (5) is solved by a fifth-order weighted essentially nonoscillatory (WENO) scheme [Shu, 1997], and the meridional components of the magnetic field are updated by $\psi$ in equation (6). In addition, special techniques in the numerical simulations of the magnetic flux rope [Hu et al., 2003; Zhang et al., 2005] are introduced here, which eliminate the numerical reconnection across the heliosphere current sheet (HCS) and guarantee the conservations of mass, axial, and toroidal magnetic fluxes of the magnetic rope.

[10] For simulations in this paper, the computational domain is taken to be $25 R_{s} \leq r \leq 300 R_{s}, 0^{\circ} \leq \theta \leq$ $180^{\circ}$ and is discretized in meshes evenly spaced with $\Delta r=$ $1.5 R_{s}$ and $\Delta \theta=1.5^{\circ}$. To avoid the complex boundary conditions associated with transonic flow, the inner boundary of computational domain is chosen so that the solar wind speed has already exceeded the fast magnetoacoustic speed. Since all waves are entering the domain at the inner boundary ( $r=25 R_{s}$ ), all quantities can be specified independently. While linear extrapolations are exerted at the outer boundary $\left(r=300 R_{s}\right)$ where all waves exit the domain, symmetric conditions are used at latitudinal directions.

\subsection{Ambient Solar Wind Equilibrium}

[11] Ambient solar wind equilibrium is obtained simply by specifying the inner boundary conditions. A unique steady state solar wind solution is obtained after $\sim 120$ hours by fixing a set of parameters at the inner boundary, with proton number density $N_{p}=550 \mathrm{~cm}^{-3}$, radial solar wind speed $v_{r}=375 \mathrm{~km}^{-1}$, magnetic field strength $B=400 \mathrm{nT}$, the plasma beta (defined as the ratio of plasma thermal to magnetic pressure) $\beta=\frac{8 \pi p}{B^{2}}=0.23$, as well as the conditions $B_{\theta}=0$ and $\mathbf{v} \| \mathbf{B}$. The configuration is quite similar to that of Wang et al. [2005], with its typical values at $25 R_{S}$ (the inner boundary) and $213 R_{S}$ (near the Earth orbit) listed in Table 1. An HCS is introduced by simply reversing the magnetic field across the equator; that is, the magnetic field directs outward (inward) in the southern (northern) semiheliosphere. Theoretically, the HCS is an ideal tangential discontinuity in MHD macroscale, but it is here smeared out over several grids by numerical diffusion. However, this slightly smeared structure is quite similar to the configuration in which an HCS is embedded in a relatively thicker heliospheric plasma sheet (HPS), which is substantiated by space observation during solar minimum [Winterhalter et al., 1994]. In addition, the equilibrium here does not resemble the bimodal nature of the solar wind with fast flow over the poles and slow flow at low latitudes. We argue that this will not distort the fundamental physical process of the MC-shock interaction, which locates mainly at low latitudes. The ambient equilibrium is described as slow solar wind astride HCS-HPS.

\subsection{Specification of MC and Shock Emergences}

[12] Specific methods for MC injection by Vandas et al. [1995] and fast shock injection by $H u$ [1998] and the methods of Hu and Jia [2001] are applied in our simulation through the inner boundary condition modification. Once the MC or shock is completely emerged into the IP medium, the original inner boundary condition as mentioned in section 2.3 is restored.

[13] The magnetic field configuration of an $\mathrm{MC}$ is described as a Lundquist solution in local cylindrical coordinates $(R, \Phi, Z)$ [Lundquist, 1950]:

$$
\left\{\begin{array}{l}
B_{R}=0 \\
B_{\Phi}=B_{0} H J_{1}(\alpha R) \\
B_{Z}=B_{0} J_{0}(\alpha R)
\end{array}\right.
$$

where $B_{0}$ specifies the magnetic field magnitude at the MC core, $H$ is the magnetic helicity, $\alpha=2.4 / R_{m}$, and $R_{m}$ is $\mathrm{MC}$ radius. With given emergence time $t_{m}$, mass $M_{m}$, speed $v_{m}$, radius $R_{m}$, plasma $\beta$, and helicity $H$, together with the above magnetic configuration, an $\mathrm{MC}$ is uniquely determined. It is unrealistic to approximate the 3-D structure of an $\mathrm{MC}$ that is rooted deeply in solar surface in a 2.5-D coordinate system. However, regarding the $\mathrm{MC}$ as a section of the 3-D magnetic loop, its dynamic characteristics could still be reflected by a $2.5-\mathrm{D}$ numerical simulation.

[14] An incidental fast shock is characterized by several parameters: its emergence time $t_{s 0}$, the latitude of its center $\theta_{s c}$, the latitudinal width of its flank $\Delta \theta_{s}$, the maximum ratio 
of total pressure (sum of thermal and magnetic pressures) at shock center $R^{*}$, the duration of growth, and maintenance and recovery phases $\left(t_{s 1}, t_{s 2}, t_{s 3}\right)$. The ratio of total pressure decreases from $R^{*}$ at the center to 1 at both flank edges as a cosine function of the angle. It varies linearly with time during the growth and recovery phases of shock disturbance. Given the upstream state at the inner boundary and
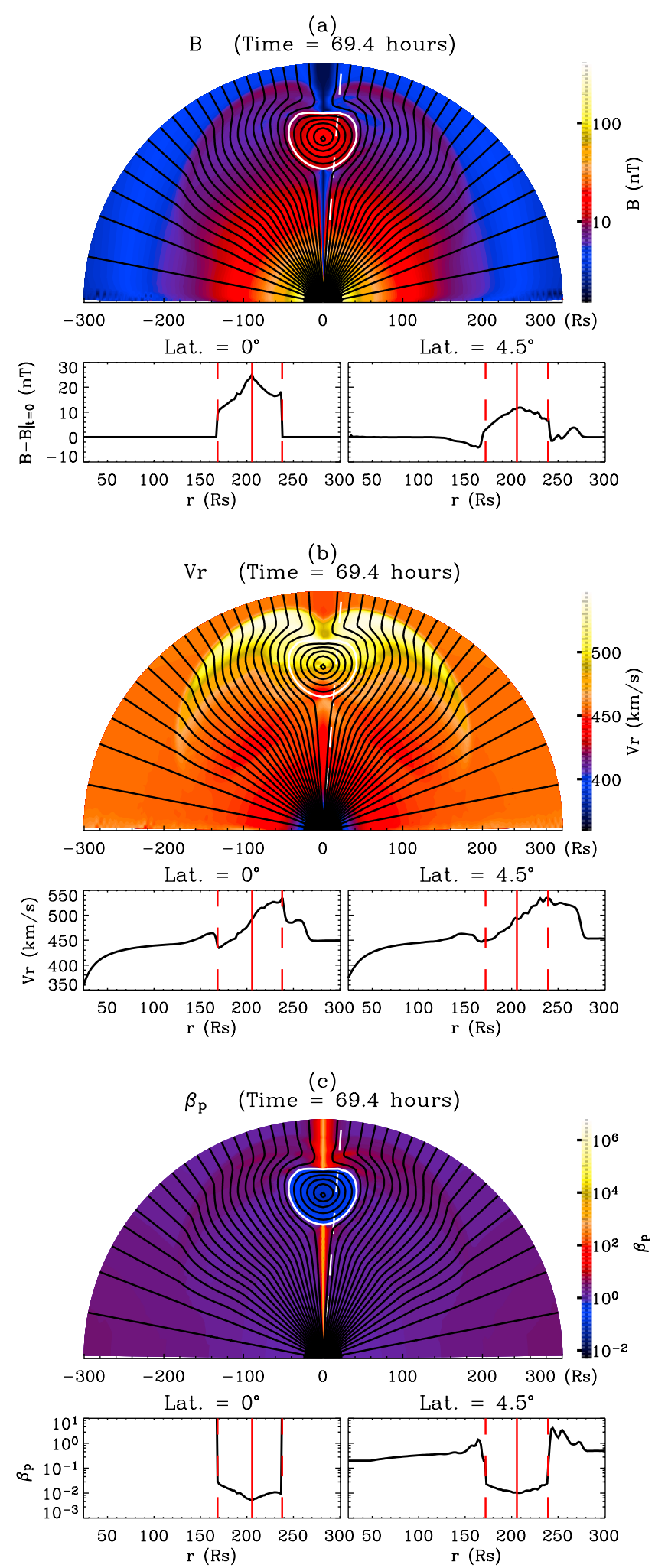

$R^{*}$, the downstream state is derived by a Rankine-Hugoniot relationship. The introduced shocks in our simulation are strong enough to be faster than the local magnetosonic speed at all times. A shock can be formed closer to the Sun, below the usually computed steady state critical points. Many solar observations show that the shock can be formed below the Alfvén critical point, which is below the inner boundary of the computational domain [e.g., Cliver et al., 2004; Raouafi et al., 2004; Cho et al., 2005].

\section{Propagation of an Individual MC (Case A)}

[15] We present here an individual MC simulation first, to manifest its characteristics as well as for comparison with the MC-shock interaction in section 4. The MC emerges along the HCS from the inner boundary. It takes the following parameters referring to equation (7),

$$
R_{m}=5 R_{s}, \quad B_{0}=1700 n T, \quad H=1
$$

and

$$
v_{m}=530 \mathrm{~km}^{-1}, \quad M_{m}=4.8 \times 10^{12} \mathrm{~kg}, \quad \beta=0.02 .
$$

Magnetic flux function $\psi$ (cf. equation (6)) is $1.51 \times$ $10^{14} \mathrm{~Wb}$ at the core of $\mathrm{MC}$, compared with $1.12 \times 10^{14}$ and $0 \mathrm{~Wb}$ in the HCS and heliospheric poles, respectively. The axial magnetic flux of MC is calculated to be $2.5 \times 10^{13} \mathrm{~Wb}$. This $\mathrm{MC}$ and its surrounding IMF have the same magnetic polarity in the meridional plane.

[16] The simulation of an MC passing near the Lagrangian point (L1) is shown in Figure 1. Under each image are two corresponding radial profiles by cutting right through $0^{\circ}$ (noted by Lat. $=0^{\circ}$ ) and $4.5^{\circ}$ (white dashed lines in the images, noted by Lat. $=4.5^{\circ}$ ) away from the equator. The magnitude of the magnetic field in radial profile is given by subtracting its corresponding initial value of ambient equilibrium. The body of the MC is identified by a white solid line in the images and between the two dotted lines in the attached profiles. The white solid line is determined by the magnetic flux function $(\psi)$ value in the equator plus a small increment. This line lies right inside the MC boundary, which has the flux function value equal to that in the equator. The $\mathrm{MC}$ core is determined by the maximum value of $\psi$. Magnetic field configuration is superimposed upon the images. As shown in Figure 1, the MC ejection into ambient solar wind results in two distinct interaction regions: (1) an MC envelope composed of IMF draping around selfenclosed MC surface and (2) a shock front and its associated

Figure 1. One snapshot of a typical MC near L1 for case A. (a) Magnetic field magnitude $B$, (b) radial flow speed $v_{r}$, and (c) proton beta $\beta_{p}$ are illustrated with two additional radial profiles along latitude $=0^{\circ}$ and $4.5^{\circ}$. Radial profile of $B$ is plotted by subtracting initial ambient value $\left.B\right|_{t}=0$. The white solid line in each image denotes the boundary of the MC. The difference of magnetic flux function $\Delta \psi$ between adjoining magnetic field lines in and out of the MC are $5.9 \times$ $10^{12}$ and $7.9 \times 10^{12} \mathrm{~Wb}$, respectively. Solid and dashed lines at each profile denote the core and boundary of MC. 


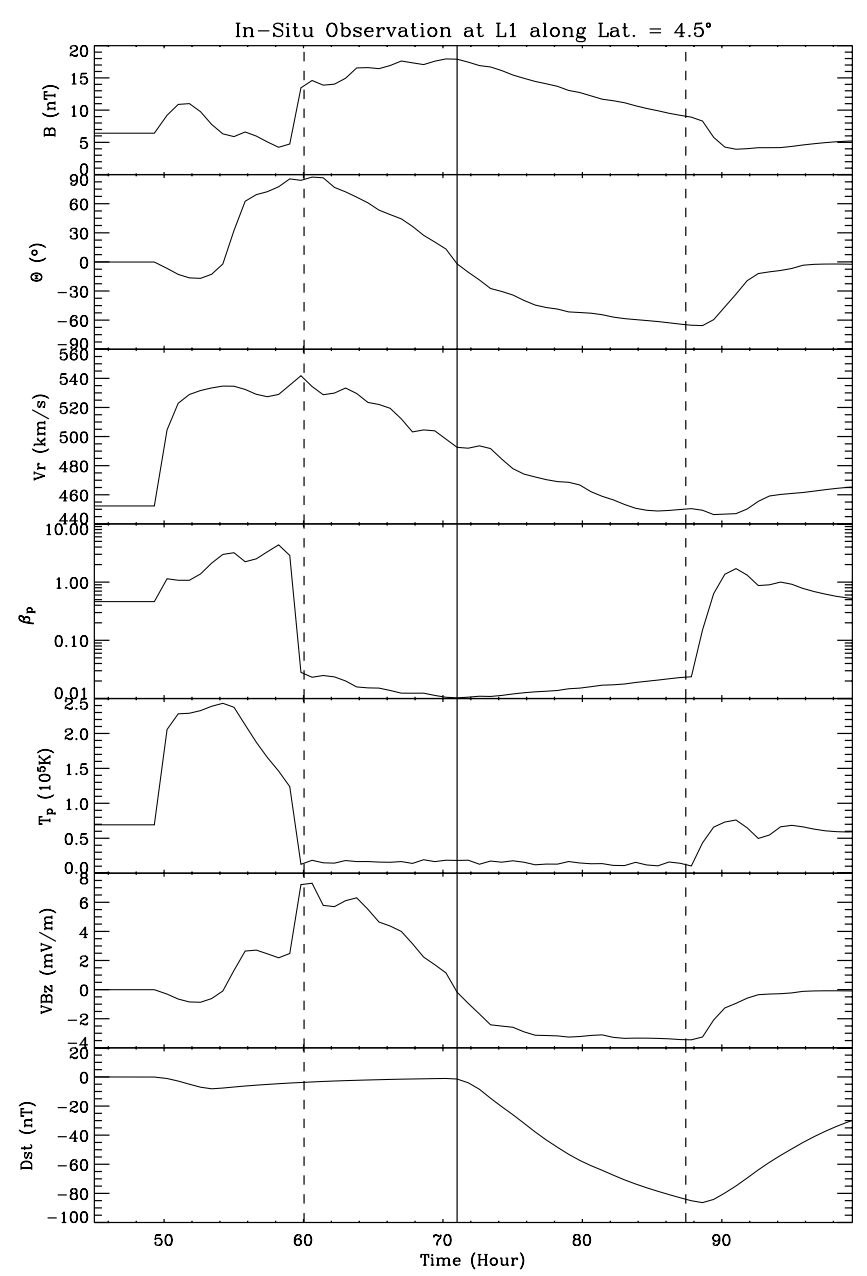

Figure 2. In situ measurements along latitude $=4.5^{\circ}$ by a hypothetical spacecraft at L1 for case A. Plotted from top to bottom are magnetic field magnitude $B$, elevation of magnetic field $\Theta$, radial flow speed $v_{r}$, proton beta $\beta_{p}$, proton temperature $T_{p}$, calculated dawn-dusk electric field $V B_{z}$, and Dst index. Solid and dashed vertical lines denote the center and boundary of MC.

sheath ahead of the MC body formed by the compression of the high-speed MC. A concave is formed at the MC-driven shock front across the HPS, as clearly seen in Figure 1b, which is also substantiated by IPS (interplanetary scintillation) observation [Watanabe et al., 1989] and shock-related simulations [Odstrčil et al., 1996a, 1996b; Hu and Jia, 2001]. The characteristics of the shock front are caused by the particular HCS-HPS structure in the heliosphere. The shock degenerates abruptly into a hydrodynamic shock because of the nearly vanishing magnetic field at the neutral current sheet. Thus the fastest and strongest shock front locates on the edges of the HPS instead of being right in the HCS. The angular width of the shock front is much larger than that of its driver-MC body. Monotonic decrease of bulk flow speed $v_{r}$ in the $\mathrm{MC}$, as seen from Figure $1 \mathrm{~b}$, implies continuous MC expansion through IP space. Moreover, many other MC characteristics are also manifested in agreement with the observations. These characteristics maintain until the MC propagates beyond the outer boundary.
[17] The in situ measurement along latitude $=4.5^{\circ}$ by a hypothetic spacecraft at L1 is shown in Figure 2. Typical MC characteristics, such as enhanced magnetic magnitude $B$ (also in the attached profiles in Figure 1a), smooth rotation of magnetic field $\Theta$, a low concave of proton temperature $T_{p}$ and proton beta $\beta_{p}$ (also in attached profiles in Figure 1c), continuous decrease of bulk flow speed $v_{r}$ (also in the attached profiles in Figure 1b), and so on, are reproduced. A sheath ahead of the MC with high temperature and high speed is clearly seen, too. The shock front and the leading, central, and trailing parts of the MC pass by L1 at 49.3, 60, 71 , and 87.4 hours successively. The MC event at L1 lasts 27.4 hours, with a maximum magnetic field magnitude (17.9 nT) and a minimum southward component (-7.7 nT). The geomagnetic effect of the simulated MC event is evaluated by the $D s t$ index, as applied by Wang et al. [2003c] using the formula $\frac{d D s t(t)}{d t}=Q(t)-\frac{D s t(t)}{\tau}[$ Burton et al., 1975], where the coupling function $Q={ }^{\tau} B_{s}$ (here $V$ is evaluated with $v_{r}, B_{s}=\min \left(B_{z}, 0\right)$, and $B_{z}$ is the $z$ component of magnetic field) and the diffusion timescale $\tau=8$ hours. The MC center approaches L1 71 hours after its departure from the inner boundary, and the value of the Dst index decreases monotonically to its minimum $-86 \mathrm{nT}$ shortly afterward (at 88.6 hours). In addition, the draping IMF within the MC-driven sheath is mainly northward. This is why the compressed magnetic field in the sheath does not cause significant Dst disturbance in our simulation.

\section{Interaction Between a Fast Shock and a Preceding MC}

\subsection{Case B}

[18] Shock compression is an efficient mechanism for the enhancement of the southward component of the magnetic field and hence serves as an IP cause of large geomagnetic storms [Wang et al., 2003b, 2003c]. The subsequent numerical simulations aim to quantify geoeffectiveness of a shock overtaking an MC in detail.

[19] To investigate the interaction between a fast forward shock and a preceding $\mathrm{MC}$, a shock centered at $\operatorname{HCS}\left(\theta_{s c}=0^{\circ}\right)$ is introduced from the inner boundary to pursue the previously occurring MC. The MC in this case is identical with that in case $\mathrm{A}$. The shock emerges at $t_{s 0}=41$ hours with its center on the equator and other parameters as follows:

$$
\begin{gathered}
\Delta \theta_{s}=6^{\circ}, \quad R^{*}=24, \quad t_{s 1}=0.3 \text { hours } \\
t_{s 2}=1 \text { hour }, \quad t_{s 3}=0.3 \text { hours. }
\end{gathered}
$$

One finds that the maximum shock speed is $1630 \mathrm{~km}^{-1}$ from the above quantities by the shock relation. The ratio of total pressure decreases from $R^{*}$ at the equator to 1 at $\pm 6^{\circ}$ aside via a cosine function. The temporal extent, as already specified, can be described as being trapezoidal as done, for example, by Smith and Dryer [1990] in the ecliptic plane.

[20] The detail process of the MC-shock event is elucidated in Figure 3. The incidental shock aphelion and the MC core arrive at $80 R_{s}$ and $155 R_{s}$, respectively, in 49.5 hours, as shown in Figures 3a, 3d, and $3 \mathrm{~g}$. The morphology of the shock front has a dimple across the HCS, similar to that of the MCdriven shock mentioned previously. In the downstream of the 

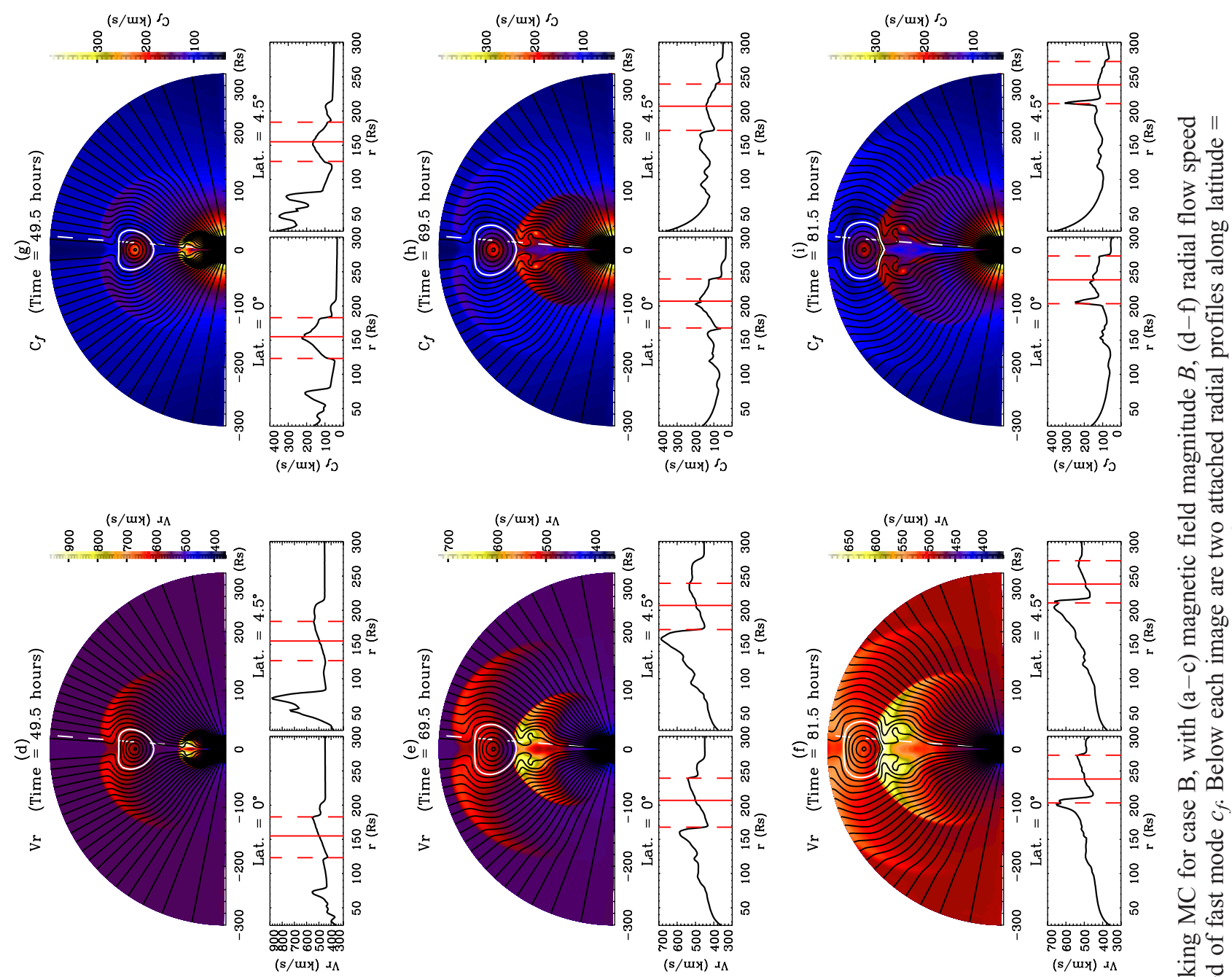

焉

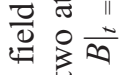

.

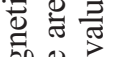

尊 800

ชิ $: \frac{0}{\square}$

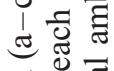

点学

$3 \div$.

ติ ต อ

\& $心$

它䓪

范焉

60, 总

면
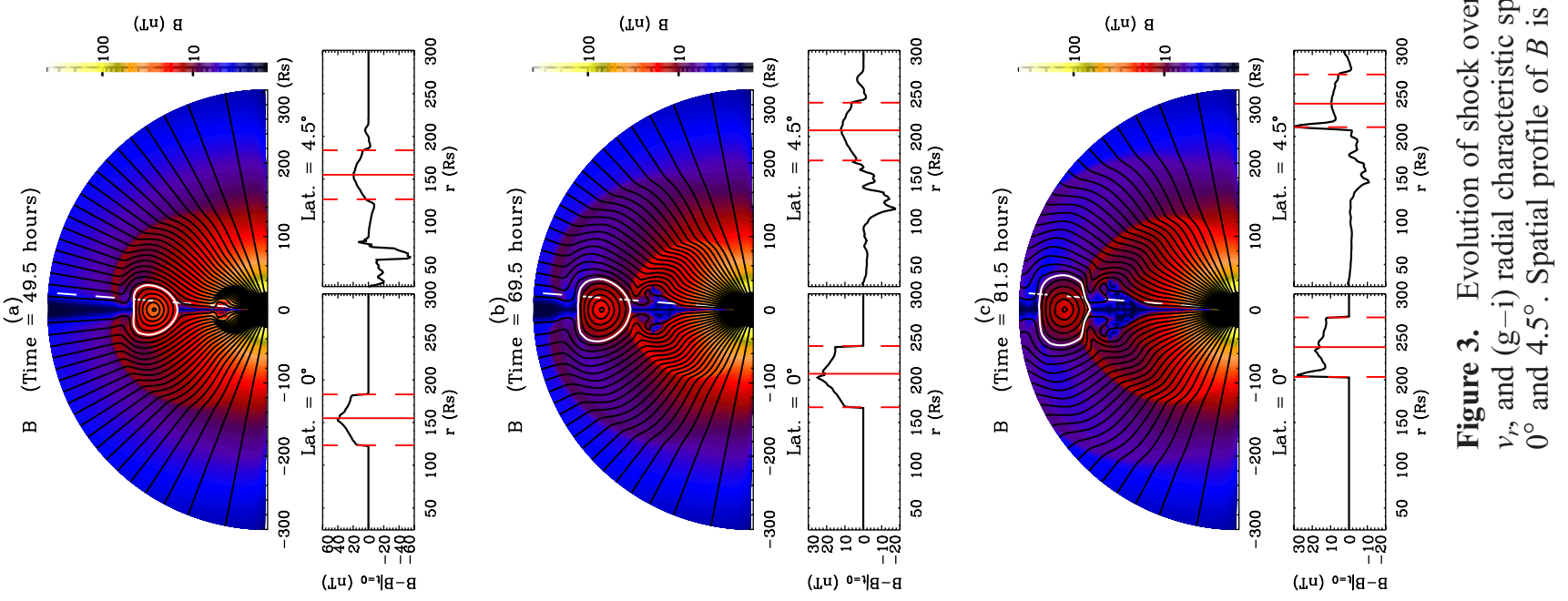


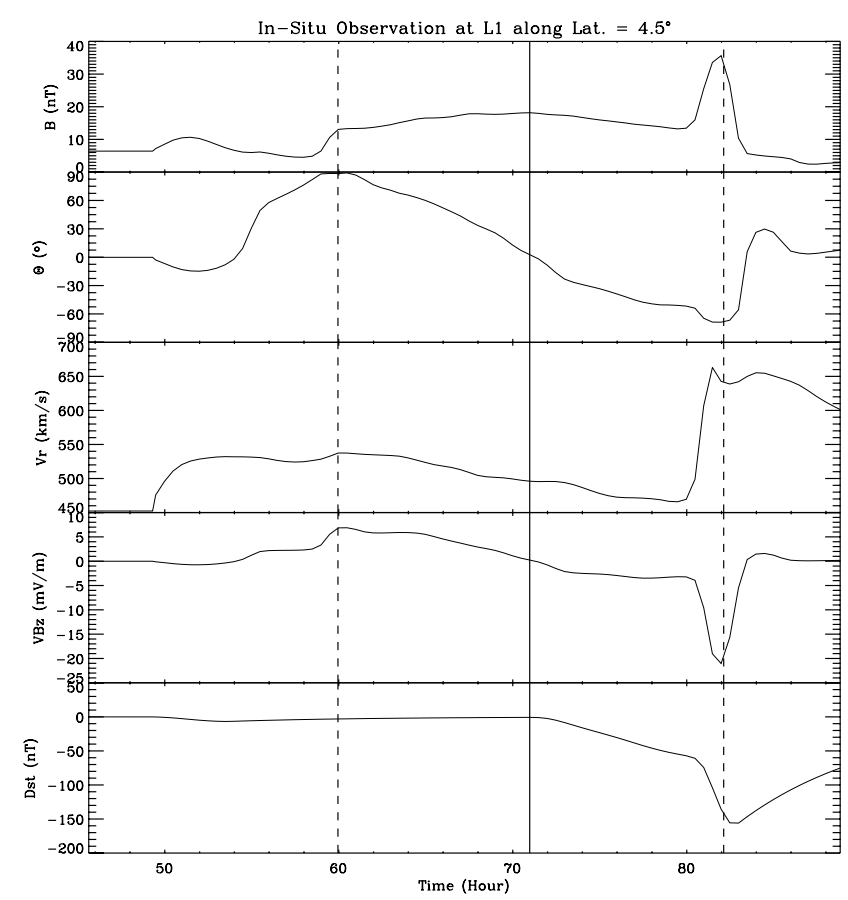

Figure 4. In situ measurements along latitude $=4.5^{\circ}$ by a hypothetic spacecraft at L1 for case B.

shock front, the flow speed reaches its maximum value, $900 \mathrm{~km}^{-1}, 4.5^{\circ}$ away from HCS, much greater than that right at the $\mathrm{HCS}$, which is $560 \mathrm{~km}^{-1}$. Comparing with the preceding $\mathrm{MC}$, which has a peak speed of $540 \mathrm{~km}^{-1}$ only, the overwhelming forward shock will soon collide with the MC body. Moreover, the tangential magnetic field component increases as the fast shock passes by, so the IMF in either semiheliosphere is deflected to the pole by the impaction of shock propagation. As a result, a "magnetic vacuum" with weaker magnetic field strength near the HCS is formed just behind the shock front, as indicated in Figures $3 a-3 c$. The shock just catches up with the inner boundary of the MC at 69.5 hours (Figures 3b, $3 \mathrm{e}$, and $3 \mathrm{~h})$. In addition, as shown in Figure $3 \mathrm{~h}$, the radial characteristic speed of the fast mode wave $c_{f}$ is very large in the $\mathrm{MC}$ body with low $\beta$. It increases steadily from $100 \mathrm{~km}^{-1}$ at the MC boundary to $200 \mathrm{~km}^{-1}$ in maximum at the MC core. There is also a peak value $180 \mathrm{~km}^{-1}$ for $c_{f}$ within the shock sheath. Meanwhile, $v_{r}$ in the MC decreases monotonically from $540 \mathrm{~km}^{-1}$ to $430 \mathrm{~km}^{-1}$ along latitude $=0^{\circ}$, as seen in Figure 3e. The MC-shock collision is pregnant at this critical time. Moreover, (1) the aphelion of the shock front locates on the edges of the HPS instead of being right at the HCS because of its concave morphology, and (2) the incidental shock in the HCS is virtually a relatively weaker hydrodynamic shock. Though the center of the shock front is along the HCS, the most violent collision can be witnessed consequently at shock aphelion rather than in the HCS when the shock and MC collide with each other. A sharp discontinuity has already been formed in the rear part of the MC at 81.5 hours (Figures 3c, 3f, and 3i). The compression along the HCS (latitude $=0^{\circ}$ ) is less significant than that along latitude $=$ $4.5^{\circ}$. The influence of the fast shock upon the MC could be reduced to two aspects: (1) enhancement of magnetic field magnitude and (2) rotation of magnetic field. As shown in Figure $3 \mathrm{c}$, the maximum value of magnetic field enhancement $\left(B-\left.B\right|_{t}=0\right)$ is $30 \mathrm{nT}$ in the compressed region, much larger than that at the $\mathrm{MC}$ core, $18 \mathrm{nT}$. Compressed magnetic field lines are very flat and point nearly southward. Both effects result in a minimum southward magnetic field $B_{z}$ with $-33 \mathrm{nT}$ at the MC tail. Furthermore, $c_{f}$ is enhanced simultaneously during shock compression, as seen from Figure 3i. In contrast with $160 \mathrm{~km}^{-1}$ at the $\mathrm{MC}$ core, $c_{f}$ at the MC tail has jumped to $300 \mathrm{~km}^{-1}$. However, the strong shock is not counteracted completely by the enhanced $c_{f}$ in the $\mathrm{MC}$ medium. Its propagation in the MC would not be stopped or diffused despite MC resistance. In addition, the domain of the so-called "magnetic vacuum" behind the shock front is magnified during the process of the shock overtaking the rear part of the MC, because the MC, an enclosed magnetic loop, serves as an obstacle in front of the shock. The MC just passes by $\mathrm{L} 1$ at 82 hours. Though the shock continues to penetrate the MC into a deeper position, the MC-shock compound structure will no longer cause the geoeffectiveness shortly after it passes by the orbit of the Earth.

[21] Similar to that in case A, the simulated data at L1 in time sequence are shown in Figure 4. A bump on the tail of the $\mathrm{MC}$ is obviously found around 81 hours, with a peak speed $660 \mathrm{~km}^{-1}$ larger than $540 \mathrm{~km}^{-1}$ at the head of the MC. As a consequence, $V B_{z}$ jumps from $-4 \mathrm{mVm}^{-1}$ to $-21 \mathrm{mVm}^{-1}$ for less than 2 hours. By comparing with Figure 2, one can see from Figure 4 that the index of geomagnetic storm Dst is $-156 \mathrm{nT}$ in the MC-shock compound structure, much greater than the $-86 \mathrm{nT}$ in the corresponding individual MC event. Moreover, the rear boundary of the MC leaves L1 5.3 hours earlier than that in case $\mathrm{A}$. The $\mathrm{MC}$ is highly compressed in its rear part by the shock.

\subsection{Case $C$}

[22] To further explore the features of the MC-shock interaction in the solar-terrestrial range, we give another case of simulation (case C) where the shock ultimately penetrates the preceding MC near L1. It is straightforward to schedule an earlier shock emergence. The shock emergence time $t_{s 0}$ is modified to be 10 hours compared with 41 hours in case B. All other parameters are the same as those in case B.

[23] Only the evolution of $v_{r}$ is given in Figure 5, to visualize the MC-shock complex structure concerned. Once the fast shock advances deeply into the $\mathrm{MC}$, the latter, superseding the ambient IP space, serves as a medium for the shock propagation. Since HCS does not exist in the MC, what ensues is the disappearance of the HCS-associated concave. The morphology of the shock front is a smooth arc in the highly compressed rear part of the MC at 20.6 hours (Figure 5b). When the shock penetrates and emerges from the MC, the HCS-HPS structure replays an important role in shock propagation. The smooth arc quickly turns into a concave across the equator with respect to the shock front at 52.1 hours, as indicated clearly by Figure $5 \mathrm{c}$. This newly emerged fast shock from the MC gradually merges with the preceding MC-driven shock into a stronger fast shock by nonlinear interaction. Moreover, sheath width, defined by 
(a)

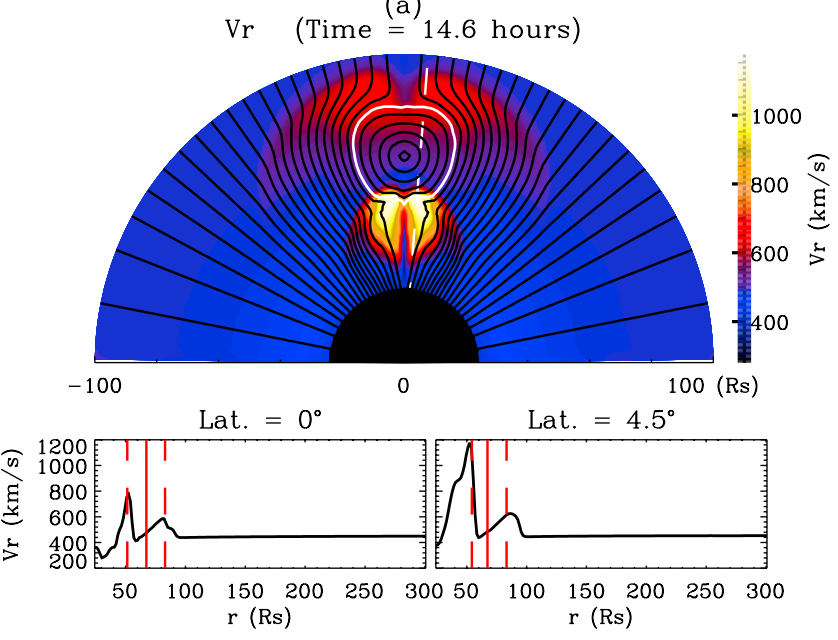

(b)
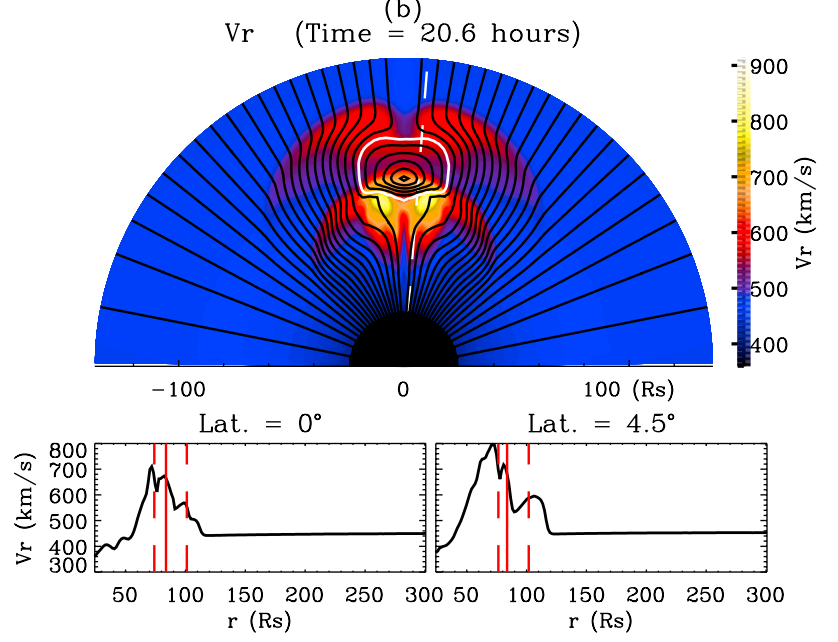

(c)

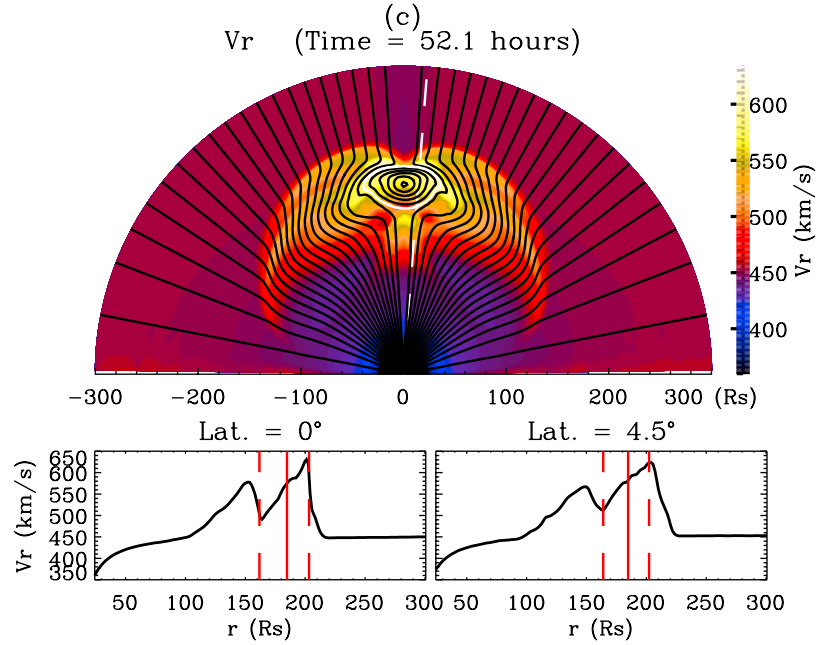

Figure 5. Evolution of shock overtaking $\mathrm{MC}$ for case $\mathrm{C}$ with radial flow speed $v_{r}$. Only part of the domain is plotted to highlight the $\mathrm{MC}$ in Figures 5a and 5b.

the radial distance along the equator between the MC-driven shock front and the outer MC boundary, is $10 R_{S}$ in case C, only half of that in case A, $20 R_{s}$. Compared with case A, several distinct differences are easily discriminated in case $\mathrm{C}$ to emphasize the shock impact: (1) the geometry of the MC boundary changes in the shape from quasi-circle to oblate ellipse; (2) the MC is highly compressed; and (3) the width of the MC-driven sheath is significantly narrowed.

[24] The hypothetical in situ measurement at L1 along latitude $=4.5^{\circ}$ is plotted in Figure 6 in contrast. The outer boundary, the center, and the inner boundary of the MC arrive at L1 at 55.5, 61, and 71.5 hours successively, which are 4.5, 10, and 15.9 hours earlier than those in case A as indicated by Figure 2. In the presence of the shock penetration, the duration of MC passing across L1 is shortened by 11.4 hours. No extremum of speed profile is found inside the MC because the shock has moved out of it. Judged only from single-spacecraft in situ observation, as seen from Figure 6, it quite likely resembles an individual $\mathrm{MC}$ event with a peak speed of $\sim 620 \mathrm{~km}^{-1}$. The greatest compression occurs at the front of the $\mathrm{MC}$, with the maximum $B=32 \mathrm{nT}$, $V B_{z}=19 \mathrm{mVm}^{-1}$, and the minimum $B_{z}=31 \mathrm{nT}$. However, the highly compressed magnetic field is northward and makes no contribution to the geomagnetic storm. This MC-shock event results in the geoeffectiveness with a value of $D s t=-107 \mathrm{nT}$.

[25] The comparison of time-dependent parameters for cases $\mathrm{A}$ and $\mathrm{C}$ is shown in Figure 7. First, the heliospheric distance of the MC core in case A depends nearly linearly on time, as shown in Figure 7a. The solar-terrestrial transporting speed of the MC core is approximately $486 \mathrm{~km}^{-1}$. It suggests that an individual MC moves at a constant speed through the IP medium, consistent with relevant simulations [Vandas et al., 1995, 1996b; Groth et al., 2000; Manchester et al., 2004a]. Meanwhile, one can see that the MC in case C is compressed by the shock, beginning from 14 hours. Second, the MC boundary is not an exact circle because of overall force balance at the $\mathrm{MC}-$ ambient flow interface. $\mathrm{MC}$

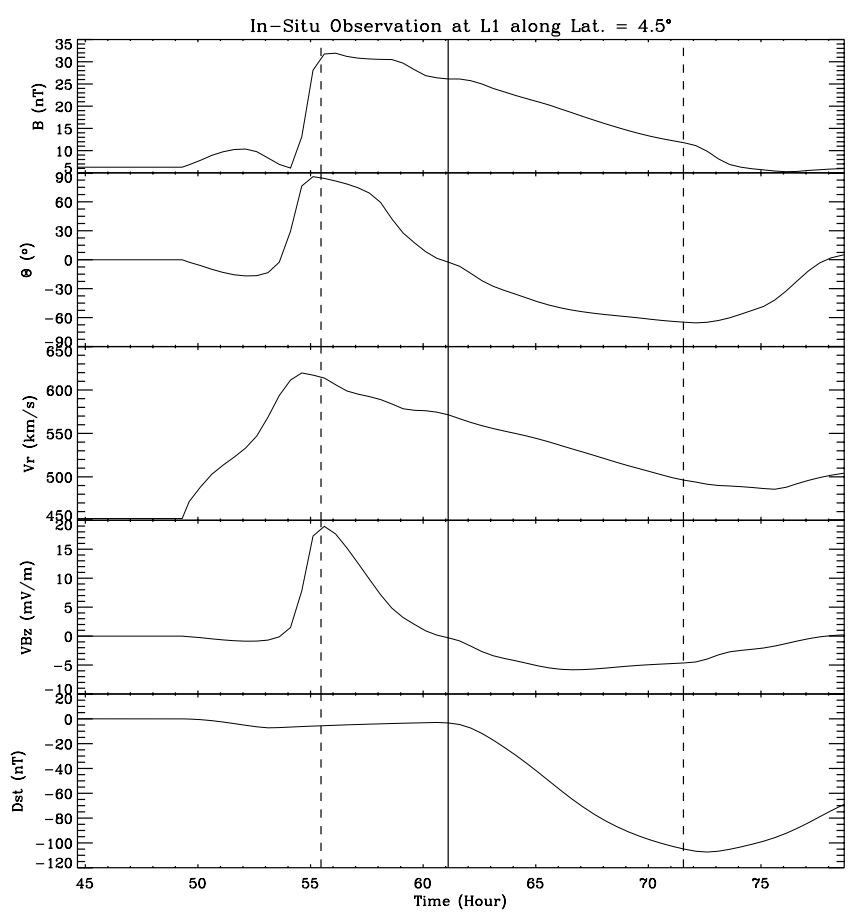

Figure 6. In situ measurements along latitude $=4.5^{\circ}$ by a hypothetical spacecraft at $\mathrm{L} 1$ for case $\mathrm{C}$. 


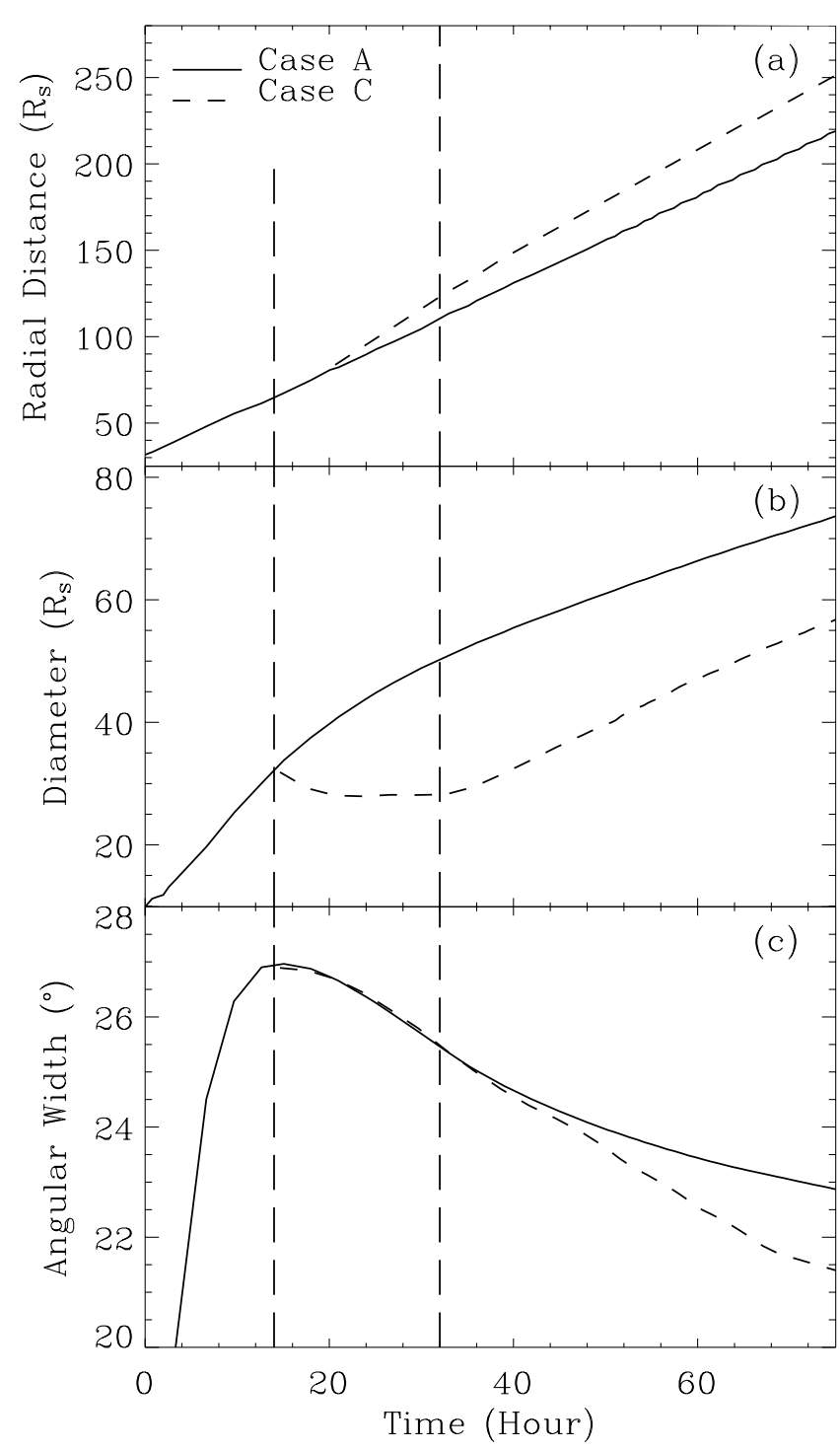

Figure 7. Time dependence of MC parameters: (a) radial distance of $\mathrm{MC}$ core, (b) $\mathrm{MC}$ diameter, and (c) $\mathrm{MC}$ angular width. The solid and dashed lines denote individual MC event (case A) and MC-shock event (case C). Two vertical dashed lines denote when the shock front arrives at the rear and front of the MC.

diameter, defined as the radial distance difference between its inner and outer boundaries along the HCS, is still used to quantify the size of the MC. One can see that the MC diameter increases monotonically to $73 R_{s}$ at $1 \mathrm{AU}$ with an asymptotic radial expansion speed $93.7 \mathrm{~km}^{-1}$ after 55 hours in case A, as indicated by Figure 7b. However, the angular width of MC in case A behaves differently, as shown in Figure 7c. It undergoes an initially rapid expansion from $15^{\circ}$ to $27^{\circ}$, then gradually recovers to $23^{\circ}$ at $1 \mathrm{AU}$. Physical interpretation for the variance of MC width is as follows: (1) The MC abruptly expands at the initial stage because its inherent magnetic field is overwhelming over that of the ambient solar wind. (2) It contracts gradually afterward while propagating in the IP medium as its magnetic field decreases faster than that of IMF. Meanwhile, the diameter and width of the MC in case $\mathrm{C}$ is compressed by the shock, as indicated in Figures $7 \mathrm{~b}$ and $7 \mathrm{c}$. Finally, the relationship between the magnetic field magnitude in $\mathrm{MC}$ core and the time in case $\mathrm{A}$ is also sought for the power $\zeta$ in $B \propto t^{-1 / \zeta}$. In our model, $\zeta$ is about 0.76 , consistent with relevant results [Vandas et al., 1995, 1996b]. The expansion of individual $\mathrm{MC}$ is pronounced from Figure 7, even on the condition of the adiabatic process $\gamma=5 / 3$. Hence our simulation is in favor of the idea that $\gamma<1$, proposed by Osherovich et al. [1993a, 1993b, 1995], may not be a strict limitation for IP MC expansion [Vandas et al., 1996b, 1996c; Vandas and Odstrčil, 2000; Skoug et al., 2000; Vandas, 2003].

[26] Moreover, the disturbance of speed enhancement just downstream of the incidental shock front cannot completely propagate into the $\mathrm{MC}$ medium. After the shock front enters the MC medium, the remaining high-speed flow follows right after the inner boundary of the preceding $\mathrm{MC}$ all the time, as seen from Figures $5 \mathrm{a}-5 \mathrm{c}$, which can also be seen in the relevant simulation [Vandas et al., 1997a, Figure 3]. The MC is highly compressed by the overtaking shock, as shown in Figure $7 \mathrm{~b}$. The MC diameter decreases monotonically during shock passage in the MC medium (14 32 hours). It then recovers gradually when the shock penetrates and emerges from the MC ( $>32$ hours). Compared to relevant simulation [Lugaz et al., 2005, Figure 7b], the behavior of the MC diameter in our simulation differs only after shock emergence from the MC medium. Because the forward shock of Lugaz et al. [2005] is driven by the following $\mathrm{MC}$, the diameter of the preceding $\mathrm{MC}$ remains constant by the compression of the following MC body when the shock propagates at the front of the preceding MC. With the push from the above-mentioned high-speed flow (Figures $5 \mathrm{a}-5 \mathrm{c}$ ) instead of the following MC body, the MC diameter cannot be completely recovered to that in the corresponding individual MC event (case A) after the passage of the fast shock.

\section{Geoeffectiveness Studies}

[27] Near-HCS latitudinal dependence of the Dst index is plotted in Figure 8. The geomagnetic storm has been obviously aggravated by the shock overtaking the MC. The minimum Dst is found to be $-103 \mathrm{nT}$ in case A, $-162 \mathrm{nT}$ in case B, and $-145 \mathrm{nT}$ in case C. In particular, the latitudinal distribution of $D s t$ in case $\mathrm{B}$ is nearly constant over latitude $=-4^{\circ} \sim 4^{\circ}$. On the one hand, the southward passing magnetic flux decreases steadily away from the equator because the MC propagates along the HCS. On the other hand, the morphology of the shock front is a concave astride the heliospheric equator when the shock penetrates into the $\mathrm{MC}$ and has just begun to change, compared with a well-established smooth arc in case C. The greatest compression occurs outside the equator. Two factors are balanced over a certain latitudinal width, thus resulting in the above-mentioned level distribution of Dst in case B.

[28] It is found from cases B and C that the geoeffectiveness of the MC-shock compound is undermined when the shock penetrates completely through the MC. To further study the dependence of Dst value on the penetration depth of the shock overtaking the MC, a set of numerical simulations with different duration between the emergence 


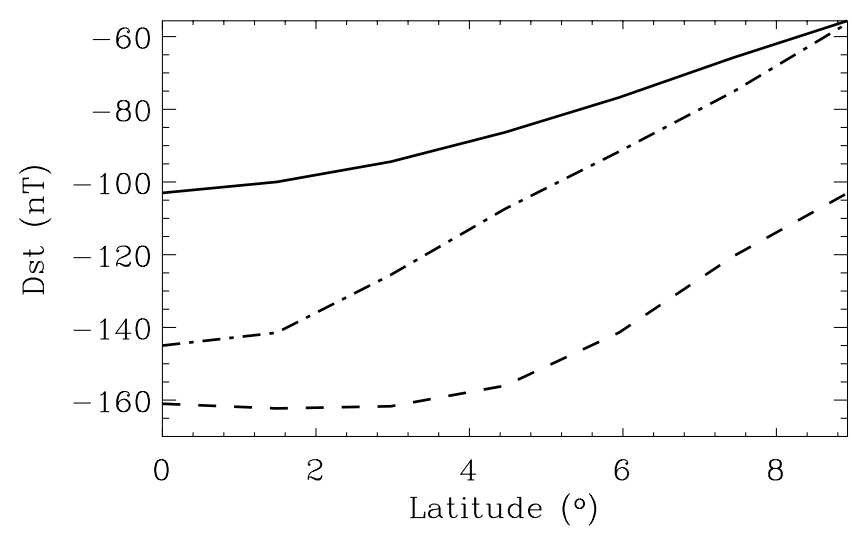

Figure 8. Comparison of latitudinal distribution of Dst index among individual MC event (case A) and MC-shock events (cases B and C). The solid, dashed, and dash-dotted lines denote cases $\mathrm{A}, \mathrm{B}$, and $\mathrm{C}$, respectively.

times of the MC and shock are carried out. Seventeen cases are run with $t_{s 0}=3,6,10,15,20,23,26,29,32,35,38,41$, $44,46,48,50$, and 60 hours.

[29] By introducing a variable $d_{D s t}$, referring to the radial distance along the heliospheric equator between the shock front and the inner boundary of the $\mathrm{MC}$, we study the timedependent data at L1 simultaneously recorded by two hypothetic spacecraft locating along latitude $=0^{\circ}$ and $4.5^{\circ}$, respectively. The geoeffectiveness of the MC-shock compound is described by Dst as an integral effect and minimum dawn-dusk electric field $V B_{z}$ as an instant effect. Synthetical analyses on some crucial parameters are given in Figure 9, where the three vertical delimiting lines (dotted, dashed, and dotted) from left to right correspond to the cases of shock encountering the tail, the core, and the front of the $\mathrm{MC}$ at $\mathrm{L} 1$, respectively. From top to bottom are

Figure 9. Parameter variances of MC-related geoeffectiveness as a function of $d_{D s t}$. Here $d_{D s t}$ refers to radial distance between the shock front and inner MC boundary along the heliospheric equator. From left to right, three vertical lines (dotted, dashed, and dotted) denote the critical situations of shock just reaching the tail, the core, and the front of the preceding $\mathrm{MC}$ at $\mathrm{L} 1$, respectively. The open triangle and the cross denote corresponding numerical results of cases $\mathrm{B}$ and $\mathrm{C}$. Shown are (a) $D t$, the duration between the emergences of $\mathrm{MC}$ and shock from the inner boundary; (b) Dst index; (c) Min. $\left(V B_{z}\right)$, the minimum of dawn-dusk electric field $V B_{z}$; (d) $\Delta t$, the interval between the commencement of $V B_{z}<-0.5 \mathrm{mV} / \mathrm{m}$ and the corresponding Dst minimum,; (e) Min. $(B s)$, the minimum of the southward magnetic component; (f) Max.(B), the maximum of magnetic magnitude; and $(\mathrm{g})$ arrival times of the $\mathrm{MC}$ and the shock along the equator. Solid and dashed lines in Figures $9 \mathrm{~b}-9 \mathrm{f}$ correspond to observations along latitude $=0^{\circ}$ and $4.5^{\circ}$. Arrival times of the outer and inner boundaries of $\mathrm{MC}$, as well as that of the incidental shock in the MC-shock events and corresponding individual shock events, are indicated by dash-dotted and dash-double-dotted lines, as well as solid and dashed lines in Figure 9g, respectively. plotted the duration between the emergences of the MC and the shock from the inner boundary, noted by $D t$ (Figure 9a); the Dst index (Figure 9b); the minimum of the dawn-dusk electric field $V B_{z}$, noted by Min. $\left(V B_{z}\right)$ (Figure $\left.9 \mathrm{c}\right)$; the interval between the commencement of $V B_{z}<-0.5 \mathrm{mV} / \mathrm{m}$ and the corresponding Dst minimum, noted by $\Delta t$ (Figure $9 \mathrm{~d}$ ); the minimum of $B_{s}$, noted by Min. $\left(B_{s}\right)$ (Figure $\left.9 \mathrm{e}\right)$; the maximum of magnetic field magnitude Max.(B) (Figure 9f); and the arrival times of the $\mathrm{MC}$ and the shock along the equator (Figure 9g). The solid and dashed lines in Figures 9b-9f correspond to the hypothetic satellites located at latitude $=0^{\circ}$ and $4.5^{\circ}$, respectively. It can be seen that the $\mathrm{MC}$ and the shock interact with each other and merge into a complex

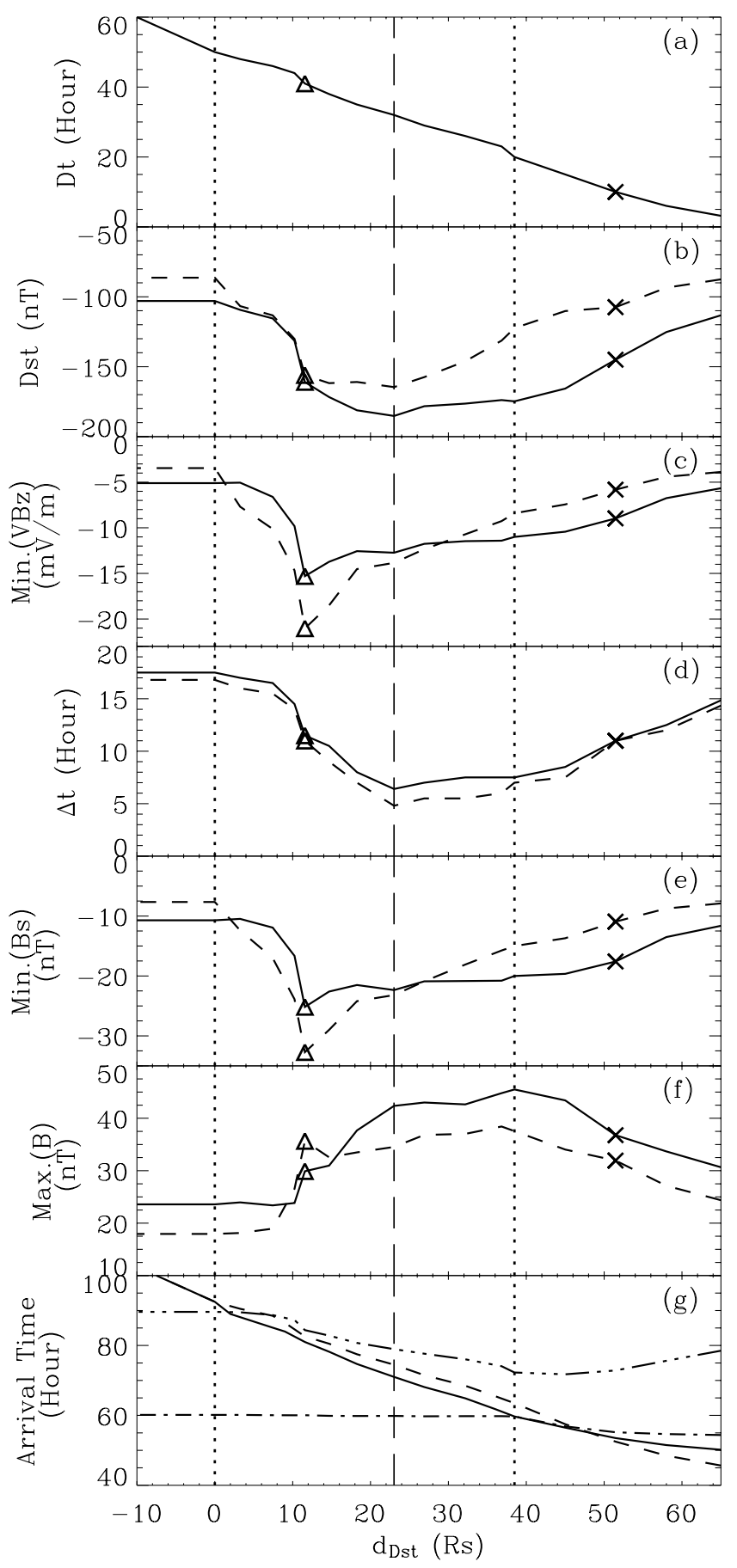


compound structure when $D t<50$ hours. The shock penetrates into the preceding $\mathrm{MC}$ more deeply with less duration between the $\mathrm{MC}$ and shock emergences. Min. $\left(B_{S}\right)$ and Min. $\left(V B_{z}\right)$ decline sharply, especially along latitude $=4.5^{\circ}$, as $d_{D s t}$ increases from 0 to $11 R_{s}$. Geoeffectiveness responses along latitude $=4.5^{\circ}$ are more dramatic because of the concave front of the incidental shock. This results in almost the same $D s t$ value over latitude $=0^{\circ} \sim 4.5^{\circ}$ for $d_{D s t}=$ $8 \sim 11 R_{s}$. Obviously, the minimum $D s t$ with $-185 \mathrm{nT}$ along latitude $=0^{\circ}$ and $-165 \mathrm{nT}$ along latitude $=4.5^{\circ}$ is obtained when the shock front just approaches the MC core at L1, corresponding to $d_{D s t}=23 R_{s}$, as indicated by the vertical dashed line of Figure 9. Moreover, Max. $(B)$ remains constant during $d_{D s t}=0 \sim 7 R_{s}$, because increasing magnetic field magnitude at the compressed region of the $\mathrm{MC}$ is not yet comparable to that at the $\mathrm{MC}$ core. Min. $\left(B_{s}\right)$ and Min. $\left(V B_{z}\right)$ are obtained at a certain position in the rear part of the MC. When the shock front exceeds the MC core and compresses its anterior part with $23 R_{s}<d_{D s t}<38.5 R_{s}$, in which the magnetic field is northward, Dst recovers in different slopes along two latitudes. It recovers gradually from -185 to $-175 \mathrm{nT}$ along latitude $=0^{\circ}$ but more rapidly from -165 to $-122 \mathrm{nT}$ along latitude $=4.5^{\circ}$. When $D t<20$ hours, the shock penetrates and propagates completely through the MC before L1. The region of dramatic interaction shifts from the MC body to the MC-driven sheath. As a result, the magnetic field tension of the MC body overcomes the grip of postshock total pressure, and Dst continues to recover monotonically as $D t$ decreases. In addition, the minimum of $\Delta t$ ( 5 hours) and that of MC passage interval (13 hours) corresponds to $d_{D s t}=$ 23 and $38.5 R_{s}$, respectively. In contrast with $-103 \mathrm{nT}$ along latitude $=0^{\circ}$ in the corresponding individual $\mathrm{MC}$ event, Dst reaches its minimum $-185 \mathrm{nT}$ along the same latitude with $80 \%$ increment in intensity when the shock front advances into the MC core. Moreover, the shock transport time in MC-shock cases is shortened within $0 R_{S}<$ $d_{D s t}<48 R_{s}$ in contrast with that in the corresponding individual shock event, as indicated in Figure $9 \mathrm{~g}$. The shortened time is 3.8 hours in maximum, corresponding to $d_{D s t}=38.5 R_{s}$. When the shock propagates from IP medium to MC medium, enhanced local magnetosonic speed and decreased bulk flow speed upstream of the shock front coexist. The joint effect of these two factors determines whether the shock is faster or slower in the MC medium. Hence the propagation speed of incidental shock influenced by the MC depends on the interval between their commencements.

\section{Concluding Remarks and Discussion}

[30] Using a 2.5-D ideal MHD numerical model, MCshock interaction and its geoeffectiveness are investigated for better understanding of the IP "shock overtaking MC" events [Wang et al., 2003b; Berdichevsky et al., 2005]. Our compound numerical algorithm is capable of capturing a sharp shock front, ensuring the absence of a magnetic monopole, guaranteeing the conservation of axial and toroidal magnetic flux of the magnetic rope, and so on. The simulations reveal dynamic characteristics of the IP MC-shock interaction and their associated geoeffectiveness in some aspects.
[31] First, numerical simulation is carried out on an individual $\mathrm{MC}$ with its inherent magnetic field overwhelming that in the ambient flow. Characteristics of the specific MC propagation through IP space are summarized as follows: (1) The MC core propagates with a nearly constant speed, and (2) its diameter expands rapidly at the initial stage. It then expands with a slower asymptotic speed; (3) its angular width also expands rapidly at the initial stage but gradually contracts afterwards. Moreover, the characteristics of an MC, such as a strong magnetic field, smooth rotation of the magnetic field, low proton temperature, low plasma $\beta$, and so on, are quite in agreement with the observations.

[32] Second, numerical simulation is conducted to model MC-shock interaction. A strong fast shock centered at the HCS emerges from the inner boundary to pursue the preceding MC. It is found that the compression and rotation of the magnetic field serve as an efficient mechanism to cause a large geomagnetic storm. The fast shock initially catches up with the preceding $\mathrm{MC}$. It then penetrates through the MC and finally merges with the MC-driven shock into a stronger compound shock. When the fast shock propagates through IP space, its front is characterized by a central concave shape in the equator; when it enters the preceding $\mathrm{MC}$, its front evolves into a purely arc shape. The morphology of the shock front is determined by the local medium. After the shock front enters the MC medium, the remaining high-speed flow just downstream of the incidental shock front cannot completely enter the preceding $\mathrm{MC}$, and it just follows behind the MC all the time. The MC is highly compressed by the overtaking shock. The solarterrestrial transport time of the incidental shock relates closely to the duration between the emergences of the MC and itself.

[33] Last, the associated geoeffectiveness is studied on the basis of numerical simulations. In contrast with the corresponding individual MC event, the MC-shock interaction results in the largest geomagnetic storm with $80 \%$ increment in terms of Dst. On the basis of an analytical solution for the process of shock propagation from the inner boundary to the center of the MC, Wang et al. [2003c] suggested that the maximum geomagnetic storm is caused by a shock penetrating the MC at a certain depth, and the stronger the incident shock is, the deeper is the position. Meanwhile, the incidental shock in our simulation is very strong, and the results show that the maximum geomagnetic storm occurs when the shock front encounters the MC core. Our numerical model agrees to some extent with that of Wang et al. [2003c]. Furthermore, the high-speed flow right after the tail of the MC boundary in our simulation mentioned previously might be responsible for the minor difference of shock penetration depth between the two models regarding the maximum geomagnetic storm.

[34] One can see that the compressed sheath field ahead of the MC in our simulations is generally northward and hence contributes little to geoeffectiveness (Figures 2, 4, and 6). If both MC helicity and ambient IMF orientation are reversed, the magnetic field within the MC-driven sheath and the front part of the MC will be directed southward and hence will be responsible for the geomagnetic storm. Some of the qualitative results compared to that discussed above can be straightforwardly conceived as follows: (1) Only when a shock propagates into the front of an MC does the 
shock exert its effect on geoeffectiveness, and (2) a shock losses its energy and momentum heavily during its propagation through the rear part of an MC so that it has relatively weaker influence on the geoeffectiveness by penetrating the preceding MC. Moreover, if an incidental shock is not strong enough, it may be dissipated quickly even in the rear part of an MC. Detailed quantitative investigation should resort to numerical simulation. This interesting topic will be addressed in the near future.

[35] Acknowledgments. We express our heartfelt thanks to the referees for their constructive comments. This work was supported by the National Natural Science Foundation of China (40274050, 40404014, 40336052, 40525014, and 40574063) and the Chinese Academy of Sciences (startup fund). M. Xiong was also supported by Innovative Fund of University of Science and Technology of China for Graduate Students (KD2005030).

[36] Zuyin Pu thanks Murray Dryer and an anonymous reviewer for their help in evaluating this paper.

\section{References}

Berdichevsky, D. B., I. G. Richardson, R. P. Lepping, and S. F. Martin (2005), On the origin and configuration of the 20 March 2003 interplanetary shock and magnetic cloud at $1 \mathrm{AU}, J$. Geophys. Res., 110, A09105, doi:10.1029/2004JA010662.

Burlaga, L. F. (1988), Magnetic clouds and force-free fields with constant alpha, J. Geophys. Res., 93, 7217.

Burlaga, L. F., E. Sittler, F. Mariani, and R. Schwenn (1981), Magnetic loop behind an interplanetary shock: Voyager, helios, and IMP 8 observations, J. Geophys. Res., 86, 6673.

Burlaga, L. F., S. P. Plunkett, and O. C. St. Cyr (2002), Successive CMEs and complex ejecta, J. Geophys. Res., 107(A10), 1266, doi:10.1029/ 2001JA000255.

Burton, R. K., R. L. McPherron, and C. T. Russell (1975), An empirical relationship between interplanetary conditions and Dst, J. Geophys. Res., $80,4204$.

Cane, H. V., I. G. Richardson, and G. Wibberenz (1997), Helios 1 and 2 observations of particle decreases, ejecta, and magnetic clouds, J. Geophys. Res., 102, 7075

Cho, K.-S., Y.-J. Moon, M. Dryer, A. Shanmugaraju, C. D. Fry, Y.-H. Kim, S.-C. Bong, and Y.-D. Park (2005), Examination of type II origin with SOHO/LASCO observations, J. Geophys. Res., 110, A12101, doi:10.1029/2004JA010744

Cliver, E. W., S. W. Kahler, and D. V. Reames (2004), Coronal shocks and solar energetic proton events, Astrophys. J., 605, 902.

Dryer, M. (1996), Comments on the origins of coronal mass ejections, Sol. Phys., 169, 421.

Dryer, M. (1998), Multidimensional magnetohydrodynamic simulation of solar-generated disturbances: Space weather forecasting of geomagnetic storms, AIAA J., 36(3), 365 .

Dryer, M., C. D. Fry, W. Sun, C. Deehr, Z. Smith, S.-I. Akasofu, and M. D. Andrews (2001), Prediction in real time of the 2000 July 14 heliospheric shock wave and its companions during the 'Bastille' epoch, Sol. Phys. 204, 267.

Dryer, M., Z. Smith, C. D. Fry, W. Sun, C. S. Deehr, and S.-I. Akasofu (2004), Real-time shock arrival predictions during the "Halloween 2003 epoch", Space Weather, 2, S09001, doi:10.1029/2004SW000087.

Farrugia, C. J., L. F. Burlaga, V. A. Osherovich, I. G. Richardson, M. P. Freeman, R. P. Lepping, and A. J. Lazarus (1993), A study of an expanding interplanatary magnetic cloud and its interaction with the Earth's magnetosphere: The interplanetary aspect, J. Geophys. Res., 98, 7621.

Fry, C. D., W. Sun, C. S. Deehr, M. Dryer, Z. Smith, S.-I. Akasofu, M. Tokumaru, and M. Kojima (2001), Improvements to the HAF solar wind model for space weather predictions, J. Geophys. Res., 106, 20,985.

Fry, C. D., M. Dryer, Z. Smith, W. Sun, C. S. Deehr, and S.-I. Akasofu (2003), Forecasting solar wind structures and shock arrival times using an ensemble of models, J. Geophys. Res., 108(A2), 1070, doi:10.1029/ 2002JA009474

Fry, C. D., M. Dryer, W. Sun, T. R. Detman, Z. K. Smith, C. S. Deehr, C.-C. Wu, S.-I. Akasofu, and D. B. Berdichevsky (2004), Solar observationbased model for multiday predictions of interplanetary shock and CME arrivals at Earth, IEEE Trans. Plasma Phys., 32(4), 1489.

Fry, C. D., M. Dryer, W. Sun, C. S. Deehr, Z. Smith, T. R. Detman, A. Aran, D. Lario, B. Sanahuja, and S.-I. Akasofu (2005), Key links in space weather: Forecasting solar-generated shocks and proton acceleration, AIAA J., 43(5), 987.
Goldstein, H. (1983), On the field configuration in magnetic clouds, in JPL Solar Wind Five, edited by M. Neugebauer, NASA Conf. Publ., 2280, 731.

Gonzalez-Esparza, A., A. Santillan, and J. Ferrer (2004), A numerical study of the interaction between two ejecta in the interplanetary medium: Oneand two-dimensional hydrodynamic simulations, Ann. Geophys., 22, 3741 .

Gosling, J. T. (1990), Coronal mass ejections and magnetic flux ropes in interplanetary space, in Physics of Magnetic Flux Ropes, Geophys. Monogr. Ser, vol. 58, edited by C. T. Russell, E. R. Priest, and L. C. Lee, p. 343, AGU, Washington, D. C.

Gosling, J. T. (1993), The solar flare myth, J. Geophys. Res., 98, 18,937. Gosling, J. T., and A. J. Hundhausen (1995), Reply, Sol. Phys., 160, 57.

Groth, C. P. T., D. L. De Zeeuw, T. I. Gombosi, and K. G. Powell (2000), Global three-dimensional MHD simulation of a space weather event: CME formation, interplanetary propagation, and interaction with the magnetosphere, J. Geophys. Res., 105, 25,053.

Harten, A. (1983), High resolution schemes for hyperbolic conservation laws, J. Comput. Phys., 49, 357.

$\mathrm{Hu}$, Y. Q. (1998), Asymmetric propagation of flare-generated shocks in the heliospheric equatorial plane, J. Geophys. Res., 103, 14,631.

$\mathrm{Hu}$, Y. Q., and X. Z. Jia (2001), Interplanetary shock interaction with the heliospheric current sheet and its associated structures, J. Geophys. Res. $106,29,299$

Hu, Y. Q., G. Q. Li, and X. Y. Xing (2003), Equilibrium and catastrophe of coronal flux ropes in axisymmetrical magnetic field, J. Geophys. Res. 108(A2), 1072, doi:10.1029/2002JA009419.

Intriligator, D. S., W. Sun, M. Dryer, C. D. Fry, C. Deehr, and J. Intriligator (2005), From the Sun to the outer heliosphere: Modeling and analyses of the interplanetary propagation of the October/November (Halloween) 2003 solar events, J. Geophys. Res., 110, A09S10, doi:10.1029/ 2004JA010939.

Jeffrey, A., and T. Taniuti (1964), Non-Linear Wave Propagation With Application to Physics and Magnetohydrodynamics, Elsevier, New York.

Klein, L. W., and L. F. Burlaga (1982), Interplanetary magnetic clouds at 1 AU, J. Geophys. Res., 87, 613.

Kumar, A., and D. M. Rust (1996), Interplanetary magnetic clouds, helicity conservation, and current-core flux-ropes, J. Geophys. Res., $101,15,667$.

Larson, D. E., et al. (1997), Tracing the topology of the October 18-20, 1995 , magnetic cloud with $\sim 0.1-10^{2} \mathrm{keV}$ electrons, Geophys. Res. Lett., $24,1911$.

Lugaz, N., W. B. Manchester IV, and T. I. Gombosi (2005), Numerical simulation of the interaction of two coronal mass ejections from Sun to Earth, Astrophys. J., 634, 651.

Lundquist, S. (1950), Magnetohydrostatic fields, Ark. Fys., 2, 361

Manchester, W. B., IV, T. I. Gombosi, I. Roussev, D. L. De Zeeuw, I. V. Sokolov, K. G. Powell, G. Tóth, and M. Opher (2004a), Three-dimensional MHD simulation of a flux rope driven CME, J. Geophys. Res., 109, A01102, doi:10.1029/2002JA009672.

Manchester, W. B., IV, T. I. Gombosi, I. Roussev, A. Ridley, D. L. De Zeeuw, I. V. Sokolov, K. G. Powell, and G. Tóth (2004b), Modeling a space weather event from the Sun to the Earth: CME generation and interplanetary propagation, J. Geophys. Res., 109, A02107, doi:10.1029/ 2003JA010150.

McKenna-Lawlor, S. M. P., M. Dryer, Z. Smith, K. Kecskemety, C. D. Fry, W. Sun, C. S. Deehr, D. Berdichevsky, K. Kudela, and G. Zastenke (2002), Arrival times of flare/halo CME associated shocks at the Earth: Comparison of the precdictions of three numerical models with these observations, Ann. Geophys., 20, 20,917.

McKenna-Lawlor, S. M. P., M. Dryer, C. D. Fry, W. Sun, D. Lario, C. S. Deehr, B. Sanahuja, V. A. Afonin, M. I. Verigin, and G. A. Kotova (2005), Predictions of energetic particle radiation in the close Martian environment, J. Geophys. Res., 110, A03102, doi:10.1029/ 2004JA010587.

Odstrčil, D., Z. Smith, and M. Dryer (1996a), Distortion of the heliospheric plasma sheet by interplanetary shocks, Geophys. Res. Lett., 23, 2521.

Odstrčil, D., M. Dryer, and Z. Smith (1996b), Propagation of an interplanetary shock along the heliospheric plasma sheet, J. Geophys. Res., 101, 19,973 .

Odstrčil, D., J. A. Linker, R. Lionello, Z. Mikic, P. Riley, V. J. Pizzo, and J. G. Luhmann (2002), Merging of coronal and heliospheric numerical two-dimensional MHD models, J. Geophys. Res., 107(A12), 1493, doi:10.1029/ 2002JA009334.

Odstrčil, D., M. Vandas, V. J. Pizzo, P. MacNeice (2003), Numerical simulation of interacting magnetic flux ropes, in Solar Wind 10, edited by M. Velli, R. Bruno, and F. Malara, AIP Conf. Proc., 679, 699.

Osherovich, V. A., and L. F. Burlaga (1997), Magnetic clouds, in Coronal Mass Ejections, Geophys. Monogr. Ser., vol. 99, edited by N. Crooker, J. A. Joselyn, and J. Feynman, p. 157, AGU, Washington, D. C. 
Osherovich, V. A., C. J. Farrugia, and L. F. Burlaga (1993a), Nonlinear evolution of magnetic flux ropes: 1. Low-beta limit, J. Geophys. Res., 98, 13,225 .

Osherovich, V. A., C. J. Farrugia, and L. F. Burlaga (1993b), Dynamics of aging magnetic clouds, Adv. Space Res., 13, 57.

Osherovich, V. A., C. J. Farrugia, and L. F. Burlaga (1995), Nonlinear evolution of magnetic flux ropes, 1. Finite-beta plasma, J. Geophys. Res., 100, 12,307.

Powell, K. G., P. L. Roe, R. S. Myong, T. Gombosi, and D. de Zeeuw (1995), An upwind scheme for magnetohydrodynamics, paper presented at 12th Computational Fluid Dynamics Conference, Am. Inst. of Aeronaut. and Astronaut., San Diego, Calif.

Raouafi, N. E., S. Mancuso, S. K. Solanki, B. Inhester, M. Mierla, G. Stenborg, J. P. Delaboudiniere, and C. Benna (2004), Shock wave driven by an expanding system of loops, Astron. Astrophys., 424, 1039.

Ryu, D., and T. W. Jones (1995), Numerical magnetohydrodynamics in astrophysics: Algorithm and tests for one-dimensional flow, Astrophys. $J ., 442,228$.

Schmidt, J. M., and P. J. Cargill (2003), Magnetic reconnection between a magnetic cloud and the solar wind magnetic field, J. Geophys. Res., 108(A1), 1023, doi:10.1029/2002JA009325.

Shu, C. W. (1997), Essentially non-oscillatory and weighted essentially non-oscillatory schemes for hyperbolic conservation laws, ICASE Rep. 97-65, Univ. Space Res. Assoc., Columbia, Md.

Skoug, R. M., W. C. Feldman, J. T. Gosling, D. J. McComas, and C. W. Smith (2000), Solar wind electron characteristics inside and outside coronal mass ejections, J. Geophys. Res., 105, 23,069.

Smart, D. F., and M. A. Shea (1985), A simplified model for timing the arrival of solar flare-initiated shocks, J. Geophys. Res., 90, 183.

Smith, Z., and M. Dryer (1990), MHD study of temporal and spatial evolution of simulated interplanetary shocks in the ecliptic plane within $1 \mathrm{AU}$, Sol. Phys., 129, 387.

Svestka, Z. (1995), On 'the solar flare myth' postulated by Gosling, Sol. Phys., 160, 53.

Vandas, M. (2003), Interplanetary modeling of ICMEs, in Proceedings of ISCS 2003 Symposium, 'Solar Variability as an Input to the Earth's Environment', edited by A. Wilson, ESA SP-535, 527.

Vandas, M., and D. Odstrčil (2000), Magnetic cloud evolution: A comparison of analytical and numerical solutions, J. Geophys. Res., 105, 12,605.

Vandas, M., and D. Odstrčil (2004), Acceleration of electrons by interacting CMEs, Astron. Astrophys., 415, 755.

Vandas, M., S. Fischer, M. Dryer, Z. Smith, and T. Detman (1995), Simulation of magnetic cloud propagation in the inner heliosphere in two dimensions: 1. A loop perpendicular to the ecliptic plane, J. Geophys. Res., $100,12,285$.

Vandas, M., S. Fischer, M. Dryer, Z. Smith, and T. Detman (1996a), Simulation of magnetic cloud propagation in the inner heliosphere in two dimensions: 2. A loop parrallel to the ecliptic plane and the role of helicity, J. Geophys. Res., 101, 2505.

Vandas, M., S. Fischer, M. Dryer, Z. Smith, and T. Detman (1996b), Parametric study of loop-like magnetic cloud propagation, J. Geophys. Res., $101,15,645$.
Vandas, M., S. Fischer, P. Pelant, M. Dryer, Z. Smith, and T. Detman (1996c), MHD simulation of the propagation of loop-like and bubblelike magnetic clouds, in Solar Wind 8, edited by D. Winterhalter et al., AIP Conf. Proc., 382, 566.

Vandas, M., S. Fischer, M. Dryer, Z. Smith, T. Detman, and A. Geranios (1997a), MHD simulation of an interaction of a shock wave with a magnetic cloud, J. Geophys. Res., 102, 22,295.

Vandas, M., S. Fischer, D. Odstrčil, M. Dryer, Z. Smith, and T. Detman (1997b), Flux ropes and spheromaks: A numerical study, in Coronal Mass Ejections, Geophys. Monogr. Ser., vol. 99, edited by N. Crooker, J. A. Joselyn, and J. Feynman, p. 169, AGU, Washington, D. C.

Vandas, M., D. Odstrčil, and S. Watari (2002), Three-dimensional MHD simulation of a loop-like magnetic cloud in the solar wind, J. Geophys. Res., 107(A9), 1236, doi:10.1029/2001JA005068.

Vassiliadis, D., A. J. Klimas, J. A. Valdivia, and D. N. Baker (1999), The Dst geomagnetic response as a function of storm phase and amplitude and the solar wind electric field, J. Geophys. Res., 104, 24,957.

Wang, Y. M., S. Wang, and P. Z. Ye (2002), Multiple magnetic clouds in interplanetary space, Sol. Phys., 211, 333.

Wang, Y. M., P. Z. Ye, and S. Wang (2003a), Multiple magnetic clouds: Several examples during March-April 2001, J. Geophys. Res., 108(A10), 1370, doi:10.1029/2003JA009850.

Wang, Y. M., P. Z. Ye, S. Wang, and X. H. Xue (2003b), An interplanetary cause of large geomagnetic storms: Fast forward shock overtaking preceding magnetic cloud, Geophys. Res. Lett., 30(13), 1700, doi:10.1029/ 2002GL016861.

Wang, Y. M., P. Z. Ye, S. Wang, and M. Xiong (2003c), Theoretical analysis on the geoeffectiveness of a shock overtaking a preceding magnetic cloud, Sol. Phys., 216, 295.

Wang, Y. M., H. N. Zheng, S. Wang, and P. Z. Ye (2005), MHD simulation on formation and propagation of multiple magnetic clouds in the heliosphere, Astron. Astrophys., 434, 309.

Watanabe, T., T. Kakinuma, M. Kojima, and R. Schwenn (1989), Largescale propagation properities of an interplanetary disturbances in association with a "halo" coronal mass ejection on 27 November 1979, Nagoya Univ. Proc. Res. Inst. Atmos., 36, 11.

Webb, D. F., T. G. Forbes, H. Aurass, J. Chen, P. Martens, B. Rompolt, V. Rusin, S. F. Martin, and V. Gaizauskas (1994), Material ejection: Report of the flares 22 workshop held at Ottawa, Canada, May 1993, Sol. Phys., 153, 73.

Winterhalter, D., E. J. Smith, M. E. Burton, and N. Murphy (1994), The heliospheric plasma sheet, J. Geophys. Res., 99, 6667.

Zhang, Y. Z., Y. Q. Hu, and J. X. Wang (2005), Double catastrophe of coronal flux rope in quadrupolar magnetic field, Astrophys. J., 626, 1096.

S. Wang, Y. Wang, M. Xiong, and H. Zheng, CAS Key Laboratory for Basic Plasma Physics, School of Earth and Space Sciences, University of Science and Technology of China, Hefei, Anhui 230026, China. (swan@ustc.edu.cn; ymwang@ustc.edu.cn; mxiong@mail.ustc.edu.cn; hue@ustc.edu.cn) 